LBL-37738

UC-404

\title{
Density Functional Theory and Pseudopotentials: A Panacea for Calculating Properties of Materials
}

\author{
M.L. Cohen \\ Department of Physics \\ University of California, Berkeley \\ and \\ Materials Sciences Division \\ Lawrence Berkeley Laboratory \\ University of California \\ Berkeley, California 94720
}

September 1995

This work was supported in part by the Director, Office of Energy Research, Office of Basic Energy Sciences, Materials Sciences Division, of the U.S. Department of Energy under Contract No. DE-AC03-76SF00098, and by the National Science Foundation under Grant No. DMR95-20554.

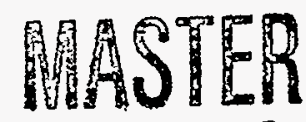

DISTRBUUTION OF THIS DOCUMENT IS UNLEMTED 


\section{DISCLAIMER}

This report was prepared as an account of work sponsored by an agency of the United States Government. Neither the United States Government nor any agency thereof, nor any of their employees, make any warranty, express or implied, or assumes any legal liability or responsibility for the accuracy, completeness, or usefulness of any information, apparatus, product, or process disclosed, or represents that its use would not infringe privately owned rights. Reference herein to any specific commercial product, process, or service by trade name, trademark, manufacturer, or otherwise does not necessarily constitute or imply its endorsement, recommendation, or favoring by the United States Government or any agency thereof. The views and opinions of authors expressed herein do not necessarily state or reflect those of the United States Government or any agency thereof. 


\section{DISCLAIMER}

Portions of this document may be illegible in electronic image products. Images are produced from the best available original document. 


\title{
Density Functional Theory and Pseudopotentials: a Panacea for Calculating Properties of Materials
}

\author{
Marvin L. Cohen \\ Department of Physics, University of California, \\ and Materials Sciences Division, Lawrence Berkeley Laboratory \\ Berkeley, California 94720
}

\begin{abstract}
Although our microscopic view of solids is still evolving, for a large class of materials one :an construct a useful first-principles or "Standard Model" of solids which is surficiently robust to explain and predict many physical properries. Both electronic and structural properties can be studied and the results of the first-principles calculations can be used to predict new materials, formulate empirical theories and simple formulae to compute material parameters, and explain trends. A discussion of the microscopic approach, applications, and empirical theories is given here, and some recent results on nanotubes, hard materials, and fullerenes are presented.
\end{abstract}

\section{Background}

The fundamental concepts tor density functional theory and for pseudopotentials were established in the 1930's by Dirac ${ }^{1}$ and Fermi. ${ }^{2}$ Much of what followed can perhaps, albeit important, be considered refinements and applications. The refinements of the basic theory have been creative and useful. In the end the objective is to achieve schemes which will provide explanations and predictions and allow close collaborations with experiment. Although it would be logical to assume that this would be the domain of the applications end of this endeavor, in fact, the feedback from experiment has greatly influenced the formalism and refinements of the theory.

In a sense this field. at least for the study of solids advanced in a manner similar to that of atomic physics. The great advances of Pauli and those who deveioped quantum theory relied on a 
deep understanding of data, in particular optical data. However unlike sharp atomic spectra, solid state spectra are broad. It is difficult to extract energy band separations without a good theoretical calculation. Just as Fermi ${ }^{2}$ introduced his pseudopotential to explain experimental data on highly excited alkali atomic levels, empirical pseudopotentials were used to decipher solid state spectra especially for semiconductors. ${ }^{3}$ These potentials proved to be transferable from compound to compound and potentials for individual atoms or pseudoatoms were obtained. ${ }^{4}$ This Empirical Pseudopotential Method 3 (EPM) not oniy allowed an interpretation of an optical or photoemission spectrum in terms of energy level separations, it also provided accurate dipole transition marrix elements. These comparisons with experiment provided security for taking wavefunctions seriously and accurate charge density plots appeared ${ }^{5}$ for the first time. Experimental verification of the calculated densities completed the program of the EPM and real space electron density plots were used to display covalent, ionic, and metallic bonding configurations and even provided quantitative results.

It was at this stage that feedback occurred to the more $\underline{a b}$ initio approaches. Not only were the standard first-principles methods not providing accurate bands and wavefunctions, the suggested directions for improvements were confusing and there was no consensus on which path was best. Now with highly accurate EPM bands, densities of states, electron densities, and response spectra, tests could be done on $\underline{a b}$ initio theories to determine their worth. This feedback proved to be as important as improvements in formalism or in computing power. An excellent example of this view is the development of Angular Resolved Photoemission Spectroscopy (ARPES) which gave the band structure $E_{n}(k)$ directly. The dramatic agreement ${ }^{6}$ between experiment and theory showed that the EPM results were accurate and the few parameters used were overdetermined with a very large margin.

An intermediate step which helped to bridge the gap between the EPM and $\underline{a b}$ initio approaches was motivated by attempts to explain properties of surfaces and interfaces. Since the EPM relied on perfect periodicity, the potentials were assumed to be unchanged at a surface, that is 


$$
V(\mathbf{r})=\sum_{\mathbf{G}} V(\mathbf{G}) S(\mathbf{G}) \mathrm{e}^{\mathrm{i} \mathbf{G} \cdot \mathbf{r}}
$$

where the structure factor $S(G)$ is an input from experiment and the form factors $V(G)$ are the parameters of the EPM (the G's are reciprocal lattice vectors). Normally only three form factors per element were necessary to fit very rich optical spectra.

To accommodate surface and interface charge redistribution, relaxation effects, and reconstruction, the EPM potential was divided ${ }^{7}$ in the usual manner into ionic and electronic potentials with exchange and correlation approximated with Slater-like $X$ - $\alpha$ parameterized approaches. Self-consistency could be achieved and surface and interface 7,8 charge redistributions could be determined. Computations were made possible by introducing the supercell concept ${ }^{9}$ so that codes based on the EPM with its inherent periodicity as demonstrated by Eq. (1), could be adapted. The self-consistent calculations gave good agreement with experiment and led naturally to the use of approaches which freed the method from the use of model pseudopotentials and $X-\alpha$ methods. In particular the use of ab initio pseudopotentials and density functional theory was the next step.

The ab initio pseudopotentials were obtained using approaches ${ }^{10-13}$ requiring only the input of the atomic number. These approaches were similar in many respects to Fermi's original method, that is by using only the outer regions of the atomic wavefunctions one is able to calculate solid-state effects. The wavefunctions are extrapolated smoothly to the core origin and these nodeless wavefunctions are then normalized. Producing potentials which yield the proper pseudowavefunctions is not difficult and many schemes are available. The earliest ${ }^{10-13}$ approaches are sometimes replaced by newer schemes having desirable attributes depending on the goals of the calculation.

In parallel with the $\underline{a b}$ initio pseudopotential developments, the density functional approach became workable 14,15 for calculations of this kind. By combining the pseudopotential and a local 
density approximation (LDA) for the density functional theory (DFT), it became possible to do precise calculations for ground state properties from $a b$ initio theory. Later developments using the "GW" approach ${ }^{16}$ allowed a consideration of excited state properties. The band gap problem which is the underestimation of the size of semiconductor and insulator band gaps by about $100 \%$ in the LDA could be overcome using the GW approach. Hence we are finally in a situation where to a good approximation it is possible to calculate ground state properties such as electronic energy structure, phonon spectra, mechanical parameters, superconductivity, and a host of other measurable properties of the ground and excited states of a wide variety of solids.

This general approach is sometimes referred to as a "standard model of solids." Unlike the standard model of particle physics, it is not a theory of everything, but it does apply for a variety of metals, semiconductors, semimetals, and insulators when the electrons are not too localized. In the following, applications are discussed to illustrate the power of the standard model and to give a perspective of the possible uses of this approach.

\section{Applications to Structure and Superconductivity}

The first goal of the researchers in this area was the determination of band structure energy levels. This, however, had been accomplished by the EPM and even today these studies give many of the most accurate values for electronic energy levels, band gaps, densities of states, and optical spectra. It was even possible to use the EPM for simple alloy and pressure calculations which made significant contributions to the field of band gap engineering. In the 1960's and 1970's when band maxima and minima were being determined, the EPM often (in fact always to our knowledge) provided the correct assignments when there were conflicts with earlier experimental determinations. Sometimes there were practical ramifications such as the assignment of the secondary conduction band minima in GaAs. These determinations were important for the Gunn effect. 
Aithough the LDA approach was known not to be appropriate for excited state properties, band structure calculations were done and the so-called band gap problem was widely discussed. At this point it is possible to obtain band structures in good agreement with the EPM by using the GW approach ${ }^{16}$ and this has become the preferred method for studies of this kind. In addition, although the charge density maps produced by the EPM are useful and provided important data for developing the ab initio approaches, it is expected that the LDA results for these maps are more accurate. Usually it's hard to determine the superior fit even when using data from several experiments.

Although the charge density plots helped researchers understand bonding trends, the standard model with $a b$ initio pseudopotentials and the LDA could be applied directly to structural problems. The first successful application which demonstrated the high precision of the method was done for $\mathrm{Si}^{17}$ and $\mathrm{Ge}^{18}$ Other materials were studied later with equal success. The calculations gave lattice constants and bulk moduli to about $1 \%$ and $5 \%$ respectively. 19 By applying a frozen-phonon approach ${ }^{19}$ it became possible to compute phonon spectra from firstprinciples. Gruneisen constants, anharmonic lattice coefficients, and a host of applications resulted from this scheme.

One of the most dramatic applications was the study of high pressure structures of Si. Although the transition from the diamond to $\beta-S \mathrm{n}$ structure at around $100 \mathrm{kB}$ was well known, transitions at higher pressure were not determined experimentally. The pseudopotential-LDA calculation for the pressure for the $\beta-S n$ transformation was $25 \%$ lower than the measured number at the time the calculations were done, but further experiment resulted in lower values and excellent agreement. In addition, all the high pressure structures predicted have been found. An additional simple hexagonal (sh) phase was found and subsequent calculations were in excellent agreement with the measurements.

The prediction of superconductivity in the sh and hcp phases was perhaps the most satisfying aspect of these calculations. After BCS theory ${ }^{20}$ was developed, a criticism was the lack 
of predictive power of this theory for new superconductors. The response of theorists at the time focused on the solution to the BCS gap equation which relied on the so-called BCS model. The result for the transition temperature $T_{c}$ in this model was one of the most famous equations in superconductivity,

$$
\mathrm{T}_{\mathrm{C}}=1.13 \mathrm{~T}_{\mathrm{D}} \mathrm{e}^{-1 / \mathrm{NV}}
$$

where $T_{D}$ is the Debye temperature, $N$ is the density of states for one spin at the Fermi energy and $\mathrm{V}$ is the effective attractive pairing potential. For a standard material like $\mathrm{Nb}$ one expects $T_{D} \sim 300 \mathrm{~K}, \mathrm{NV} \sim 0.3$, and $T_{c} \sim 11 \mathrm{~K}$. However, the exponential sensitivity to $\mathrm{NV}$ for this range of parameters causes problems for first principles calculations. If NV is reduced by a factor of 10 , which is not unlikely when one is balancing the attractive phonon induced pairing potential against the repulsive Coulomb potential, $T_{c}$ becomes of order $10^{-12} \mathrm{~K}$. This demonstration was commonly used by theorists to show how difficult the prediction of new superconductors would be. It was not a problem with the superconductivity theory which was limiting the precision but rather the knowledge of the normal state was insufficient to do a credible calculation of $T_{c}$. Since both sh and hcp Si phases are metallic and the electronic and vibrational spectra could be calculated from first principles, these phases proved to be ideal for studying the possibility of ab initio calculations of superconducting properties. Another motivation was the observation that the charge density maps demonstrated that these metals are highly covalent and it had been proposed 21 that covalency in metallic systems was favorable for superconductivity.

The electron-phonon parameter was calculated and the Coulomb repulsion parameter was obtained using a scaling plot which depends only on $\mathrm{N}$. This parameter is relatively constant for superconductors. Hence in principle, except for the atomic number and atomic mass (to determine the phonon spectra), the only input to this calculation was the structures (sh and hcp) and the density of states $\mathrm{N}$ to obtain the Coulomb parameter. The result ${ }^{22}$ was a successful prediction of superconductivity and the dependence of $T_{c}$ on pressure. The fact that the material's existence; its 
structural, electronic and vibrational properties; and even its superconducting properties could be determined from first principles added considerable credibility for the standard model.

\section{Hardness/Bulk Moduli}

Although the goal of many calculations of material properties done by theoretical physicists and chemists is to do first-principles calculations, there is a great benefit in using semi-empirical models derived from the first principles approaches. The EPM is such a scheme. Another is a semi-empirical approach for determining the bulk modulus B of a material. A semi-empirical theory of B is not needed if one is only interested in a single material since the total energy pseudopotential-LDA approach gives accurate values for $B$. However, if one is interested in trends for materials and in finding what physical parameters determine $B$, a semi-empirical approach is very useful.

Using the Phillips-Van Vechten spectral theory of semiconductors ${ }^{23}$ it is possible to derive 24 a scaling relationship between $B$ and the bond length $d$ for covalent valence 8 systems. One can add the effects of non-tetrahedral coordination with an empirical factor $N_{c} / 4$ where $N_{c}$ is the coordination number. Because ionicity reduces $B$, a simple empirical adjustment uses $I=0,1,2$ for group $\mathrm{IV}, \mathrm{III}-\mathrm{V}$, and II-VI semiconductors in the expression

$$
B=\frac{N_{c}}{4} \frac{1971-220 I}{d^{3.5}}
$$

where $\mathrm{B}$ is given in GPa when the measured $d$ is expressed in Angstroms. The agreement with experiment and Eq.(3) for standard diamond, zincblende, and wurtzite semiconductors and insulators is excellent. 25 The results are as good as the first principles calculations, that is, the largest errors are of the order of $5 \%$.

One obvious conclusion to be drawn from Eq.(3) is that B is maximum when $d$ and $I$ are minimized. The dependence on $\mathrm{d}$ is the most crucial and this suggests the exploration of compounds formed from elements in the first row of the periodic table. The most studied materials 
in this group are diamond and BN. It was satisfying to find that the semi-empirical equation correctly predicted $\mathrm{B}$ for $\mathrm{BN}$ when a direct experimental ${ }^{26}$ measurement was done. Earlier indirect measurements were inconsistent with the results of Eq.(3).

A motivation for finding large values of $B$ comparable or greater than that of diamond is to suggest possible synthesis of superhard materials. Although hardness is a macroscopic property depending on defects, impurities, dislocations, and shear moduli, there is often a strong correlation between hardness and B. Assuming this correlation to be correct, an investigation of possible large B materials is likely to lead to superhard materials. In fact, assuming that Eq.(3) is applicable, there appears to be no natural limit or cutoff on $B$ in the range of $d$ we are considering. Investigation ${ }^{24}$ of atomic sizes for first row atoms suggests that the $\mathrm{C}-\mathrm{N}$ bond can be shorter than the $\mathrm{C}-\mathrm{C}$ bond in an $\mathrm{sp}^{3}$ configuration. Hence based on the $\mathrm{B}(\mathrm{d})$ dependence of Eq.(3) it was suggested 24 that solids composed of $\mathrm{C}$ and $\mathrm{N}$ might yield $\mathrm{B}$ 's comparable to or exceeding that of diamond.

Although a candidate structure for $\mathrm{C}-\mathrm{N}$ is zincblende, without defects it is probably unlikely that this structure is suitable because if a single $\mathrm{CN}$ molecule is assumed in each cell, this nine electron system is likely to be unstable since it will allow occupancy of antibonding conduction band states. Hence, it was suggested 27 that $\beta-C_{3} N_{4}$, which is $C-N$ in the $\beta-S_{3} N_{4}$ structure, might be stable, and a first principles total energy calculation 27 gave results for B which are consistent with estimates from Eq.(3). Some other candidate structures have been explored,28 but there does not appear to be a consensus on a candidate structure which is much more likely than the $\beta-C_{3} N_{4}$ proposal. Of course, one cannot rule out the possibility of more stable structures. In fact this is likely.

A series of experimental searches have been undertaken. Some representative results are given in references 29-34. In general the experimental situation is encouraging, however large samples are not available for indentor tests although the hardness of some films have been examined. From a theoretical point of view these studies suggest that it may be possible that the 
buik modulus of diamond can be exceeded and that nitrogen implants in diamond should toughen surfaces.

There are other interesing properties expected for $\beta-C_{3} \mathrm{~N}_{4}$. Because of the small masses of the first-row elements in this compound it is expected that the thermal conductivity of this material should be very large. Another property of interest is the energy band gap. A complete LDA calculation for the band structure $E_{n}(k)$ has been performed 35 which yields a semiconductor system with an indirect band gap and a direct gap at slightly higher energy. By using a GW approach $^{35}$ to examine the band gaps, the resulting values for the indirect and direct gaps are $6.4 \mathrm{eV}$ and $6.75 \mathrm{eV}$ respectively.

Hence, if produced, $\beta-C_{3} N_{4}$ could be a userul material because of severai or its predicted properties: high bulk modulus, hardness, high thermal conductivity, and large band gap insulator.

\section{Applications to Fullerenes}

The discovery of the $\mathrm{C}_{60}$ molecule 36 and the subsequent intense research on $\mathrm{M}_{3} \mathrm{C}_{60}$ where $M$ is an alkali metal have added to the current excitement in materials science. These systems behave in interesting ways; they are superconductors, ${ }^{37}$ and there has been considerable discussion of whether standard theory is applicable. In particular, because of the narrow band widths expected for these solids, the quesrion of whether the standard model described earlier is applicable. Correlation effects are expected, however it is the size of their influence which is relevant. Another important theoretical question is whether the BCS theory with electron pairing arising from phonon exchange is the appropriate description of these exoric systems.

Although this field is still maturing, it appears that the answers to the questions of the appropriateness of band theory and of BCS theory is mostly affirmative. Calculations of the band structure based on the LDA gives the usual underestimation of the band gap, however when the GW corrections are included, good agreement for the gap and with the photoemission data is 
obtained. 38 There are correlation effects which are not negligible, however the main features of the bands calculated with the standard model appear to be correct.

It also appears that it is possible to obtain a consistent picture ${ }^{39}$ of the superconductivity in the $\mathrm{M}_{3} \mathrm{C}_{60}$ systems within $\mathrm{BCS}$ with a phonon pairing mechanism. In particular, the phonons which seem to dominate in this model are those associated with intramolecular excitations. If a McMillan ${ }^{40}$ type equation is used instead of Eq.(2) then

$$
T_{C} \sim T_{D} e^{-\frac{1}{\lambda^{*}-\mu^{*}}}
$$

where $\lambda^{*}=\frac{\lambda}{1+\lambda}$ and $\mu^{*}=\frac{\mu}{1+\mu \ell n \frac{T_{D}}{T_{F}}}$. The electron-phonon parameter $\lambda$ and its Coulomb counterpart $\mu$ are essentially the components of NV in the BCS model equation (2), where NV= $\lambda$ $\mu$. The $\lambda *$ parameter is the result of renormalization and $\mu^{*}$ arises because of the different energy scales for the phonon and Coulomb interactions, that is the Debye temperature $T_{D}$ and the Fermi temperature $T_{F}$.

It has been argued that for the fullerenes the reduction of $\mu$ to $\mu^{*}$ which is characteristically from values near 0.4 to 0.1 will not occur because of the larger than usual ratio of $\mathrm{TD} / \mathrm{TF}$. In addition, because of the relatively large density of states in these systems, a fairly large $\mu$ is expected. One can argue, however, that the large densiry of states which appears as a multiplicative factor along with the Coulomb potential is expected to be cancelled to a fairly large extent by the screening of the electron gas. This is easiest to show in a simple Thomas-Fermi model where the screening wavevector $\mathrm{K}_{S}^{2}$ is proportional to the density of states. Hence for large values of $\mathrm{N}$, there is almost complete cancellation in this model. A more first-principles calculation has been done ${ }^{41}$ for $\mu^{*}$ and the results support the arguments given above. The resulting $\mu^{*}$ is in the range of $\sim 0.2$ which is a "manageable" value.

The question of contributions to $\lambda$ from phonon induced pairing has been examined by various authors $42-44$ and their results differ primarily in which phonons contribute to $\lambda$. 
Analysis ${ }^{45}$ of normal state resistivity data allows some possibility for discerning between the candidates. One physical argument for concluding that intramolecular vibrations dominate is the dependence of the superconducting transition temperature $T_{c}$ on pressure $P$. The fullerene superconductors behave oppositely to conventional superconductors like $A l$ since $\frac{d T_{c}}{d P}<0$. Since $\lambda$ has a multiplicative density of states factor which decreases with pressure and since intramolecular phonons should be relatively uninfluenced by pressure, the result is a decrease in $\lambda$ with pressure and hence a decrease in $T_{\mathfrak{c}}$. A similar but inverse effect is seen when the lattice constant is increased because of the use of large alkalis.

Hence at this point there appears to be a consistent picture of both the electronic structure and the superconductivity for these systems. Band theory within the standard model appears adequate to explain the electronic properties and the details of the superconducting properties seem to be consistent with BCS theory when material properties are considered. In some sense the successful application of the standard model to fullerene properties demonstrates the robustness of this theory. However, some calculations such as the electronic structure may require additional theoretical models to account for the expected correlation effects in these systems.

\section{Applications to Nanotubes}

The discovery ${ }^{16}$ of carbon nanotubes (bucky tubes) further illustrates the richness of carbon chemistry. In these systems, single wall or concentric carbon tubes with radii on a nanometer scale can be viewed as rolled up graphite sheets. The electronic properties of these tubes are predicted 47 to change with the chirality introduced when the sheet is rolled into a tube. Semiconductor and semimetal systems can be obtained with purely geomerric changes in the tubes-without the necessity or doping. At present the primary experimental tool for investigating these systems is electron microscopy. Contacts to the rubes are difficult to make, but the siruation is improving. 
A particularly interesting question is the study of the nature of the electronic properties of the tubes when they are filled with atoms such as $\mathrm{K}$. Calculations 48 indicate that there should be significant charge transfer from the $\mathrm{K}$ atoms to the inter tube walls. Total energy calculations ${ }^{48}$ comparing the energy of an empty tube and a tube filled with a linear arrangement of $\mathrm{K}$ atoms suggest a lower energy for the latter. To achieve this situation experimentally it will be necessary to grow tubes with open ends and/or open the ends of closed tubes. Recent experiment with tubes grown in a hydrogen atmosphere indicate the $\mathrm{H}$ atoms may cap dangling bonds resulting in tubes with open ends. Hence the introduction of $\mathrm{K}$ or other atoms may be possible with tubes grown in this way. Questions relating to the possible metal conductivity or even the superconductivity 49 of such system are interesting and perhaps answerable experimentally.

A new area in the study of nanotubes involves compound systems based on $\mathrm{B}_{\mathrm{x}} \mathrm{C}_{\mathrm{y}} \mathrm{N}_{\mathrm{z}}$. Unlike $\mathrm{C}_{60}$ analogues with $\mathrm{BN}$ where structural frustration does not allow the molecule to form, $\mathrm{BN}$ graphitic sheets are known to exist and the prediction ${ }^{50}$ of $\mathrm{BN}$ nanotubes has been verified experimentally. 51 Unlike the $C$ nanotube case all $\mathrm{BN}$ tubes are expected to be semiconducting 52 with fairly large band gaps because of the ionic nature of this system. Other interesting ionic compounds are $\mathrm{BC}_{2} \mathrm{~N}$ and $\mathrm{BC}_{3}$. Both materials exist in layer like structures and calculations 53,54 indicate that tubule forms should be stable. For $\mathrm{BC}_{2} \mathrm{~N}$, it can be shown ${ }^{53}$ that the anisotropic conductivity of this material leads to chiral currents when the tube is formed. This raises the possibility of creating nanocoils based on $\mathrm{BC}_{2} \mathrm{~N}$. The predicted ${ }^{54}$ properties of $\mathrm{BC}_{3}$ are also novel. Single tubes are expected to be semiconductors while bunches of tubes should exhibit metallic conductivity because of tube-tube interactions.

The doping of nanotubes adds significantly to the range of properties which can be explored; however, it is interesting to focus on the unusual possibilities of varying the electronic properties of tubules by altering their structure. The caps on the ends of tubes and the shape of the narrowing down of tubes near their ends require different rings of atoms other than 6 . Using $C$ as a prototype tubule, posirive curvarure can be obtained, as in the $\mathrm{C}_{60}$ molecule, by introducing 
5-fold rings. Negative curvature is achieved with 7-fold rings and 4-fold and 8-fold rings can cause other changes in the tubule curvatures.

Introducing rings other than 6-fold rings not only changes the geometric structure and structural properties, it also alters the electronic properties of a tube. An interesting example arises when two 6-fold rings are replaced by a 5 fold-7 fold "defect." This defect allows 55 the joining of a chiral tube and non-chiral tube. The result is a nano-heterojunction with two semiconductors of different band gaps joined by a small "interface" created by the "5-7" defect. In a similar manner Schottky barriers can be formed. These studies may make nanodevices possible where the scale is just about as small as one can picture when using groups of atoms.

The 5-7 defect raises the question of whether this variety of ring geometry can be applied more generally. It is in fact possible 56 to produce a periodic unit cell based on 5 -fold and 7 -fold rings which will cover all space just as the 6-fold rings of $C$ atoms in graphite sheets do. However, unlike graphite sheets which are semimetal-like with a very small density of states at the Fermi energy $E_{F}$ the 5-7 arrangement yields a metallic sheet with a much larger density of states at E.

Simple geometric considerations provided the initial structural coordinates for both the $(10,0)-(9,1)$ heterostructure Fig. 1 and the planar 5-7 network Fig. 2. These crude initial structures were relaxed with tight-binding total energy molecular dynamics ${ }^{5 i}$ over roughly three hundred time steps of 30 femtoseconds each. The $(10,0)-(9,1)$ heterostructure was simulated in a 248-atom supercell with tube ends sufficiently far from the defect region that the dangling bonds had no discernible effect on the local structure of the defect. The planar C-57 nerwork was initially simulated as a finite 141-atom sheet. After initial relaxation, the coordinates were randomly perturbed by $\sim 0.1 \AA$ each and the structure was annealed at $2000 \mathrm{~K}$. The structure remained planar to within $0.01 \AA$. After annealing and relaxation, unit cell coordinates were extracted from the atoms nearest the center of the sheet. Comparison of several different unit cells near the sheet center indicated that errors due to the finite size of the sheet did not exceed $0.01 \AA$. These unit cell 
coordinated were then used for a tight-binding total energy calcuiation of the periodic structure. This calculation indicated that 5-7 carbon is a metal with a density of states at the Fermi level of 0.1 states per $\mathrm{eV}$ per atom. In addition, the cohesive energy obtained for the $5-7$ network is $\sim 0.3 \mathrm{eV}$ per atom above that calculated for graphite. For comparison, $\mathrm{C}_{60}$ has a total energy roughly $\sim 0.4 \mathrm{eV}$ per atom above that of graphite within the tight binding total energy formalism. Assuming that a suitable synthetic pathway can be found, planar 5-7 carbon should be a new metastable state of carbon, and the only known crystalline pure-carbon metal.

This area of research is new and it appears to be fertile. It is fortunate that the standard model is sufficiently robust to allow structural and electronic calculations. Close collaboration between experiment and theory can be very useful in this emerging field.

\section{Conclusions}

The theme of this paper has been on the applications of pseudopotential theory and density functional theory what can be described as a standard model of solids. Applications to structural problems such as pressure induced solid-solid phase transitions add to the credibility of this approach. Lattice constants, bulk moduli, elastic constants, vibrational spectra, electron-phonon interactions and a host of other ground state properties can be determined just using the LDA. Excited state properties such as band gaps and response functions for optical probes can be evaluated using a GW extension of the LDA. Areas such as superconductivity, $\mathrm{C}_{60}$ systems, nanotubes, and others have benefited from this robust theory. Further adjustments and additions to the theory are likely, but it is probably wise to let experiment dictate the need for changes along with the natural development of the formalism of DFT and pseudopotentials or electronic structure in general. Another useful tool which has paved the way for new developments is the use of semiempirical models. Not only do these approaches help to explain and predict properties of materials, they also often point the way for the future developments of the $\underline{a b}$ initio theories.

\section{Acknowledgments}


I would like to acknowledge contriburions from Dr. V.H. Crespi.

This work was supported by National Science Foundation Grant No. DMR-9520554 and by the Director, Office of Energy Research, Office of Basic Energy Sciences, Materials Sciences Division of the U. S. Department of Energy under Contract No. DE-AC03-76SF00098.

\section{References}

1. P.A.M. Dirac, Proc. Cambridge Philos. Soc. 26, 376 (1930).

2. E. Fermi, Nuovo Cimento 11, 157 (1934).

3. M. L. Cohen and J. R. Chelikowsky, Electronic Structure and Optical Properties of Semiconductors (Springer-Verlag, Berlin, 1988).

4. M. L. Cohen and V. Heine, in Solid State Physics, Vol. 24, eds. H. Ehrenreich. F. Seitz, and D. Turnbull (Academic Press, New York, 1970), p.37.

5. J. P. Walter and M. L. Cohen, Phys. Rev. Lett. 26, 17 (1971).

6. T.C. Chiang, J.A. Knapp, M. Aono, and D.E. Eastman, Phys. Rev. B 21, 3513 (1980).

7. M. Schlüter, J. R. Chelikowsky, S. G. Louie, and M. L. Cohen, Phys. Rev. B 12, 4200 (1975).

8. M. L. Cohen, in Advances in Electronics and Electron Physics, Vol. 51, eds. L. Marton and C. Marton (Academic Press, New York, 1980), p.1.

9. M. L. Cohen, M. Schlüter, J. R. Chelikowsky, and S. G. Louie, Phys. Rev. B' 12, 5575 (1975).

10. T. Stafkloff and J.D. Joannopoulos. Phys. Rev. B 19, 1077 (1979).

11. G.P. Kerker, J. Phys. C 13, L189 (1980).

12. A. Zunger and M. L. Cohen. Phys. Rev. B 18, 5449 (1978).

13. D.R. Hamann. M. Schlütter, and C. Chiang, Phys. Rev. Lett. 43, 1494 (1979).

14. P. Hohenberg and W. Kohn, Phys. Rev. B 136. $S 64$ (1964).

15. W. Kohn and L.J. Sham, Phys. Rev. A 140, 1133 (1965).

16. M. Hybertsen and S.G. Louie, Phys. Rev. Lett. 55, 1418 (1985).

17. M. T. Yin and M. L. Cohen, Phys. Rev. Lett. 45, 1004 (1980).

18. M. T. Yin and M. L. Cohen, Phys. Rev. B 26, 5668 (1982). 
19. M. L. Cohen, Physica Scripta T1, 5 (1982).

20. J. Bardeen, L.N. Cooper, and J.R. Schrieffer, Phys. Rev. 106, 162 (1957).

21. M. L. Cohen and P. W. Anderson, in Superconductivity in d-and f-band Metals, ed. D. H. Douglass (AIP, New York, 1972), p.17.

22. K. J. Chang, M. M. Dacorogna, M. L. Cohen, J. M. Mignot, G. Chouteau, and G. Martinez, Phys. Rev. Lett. 54, 2375 (1985).

23. J. Phillips, "Bonds and Bands in Semiconductors," (Academic Press, New York, 1973).

24. M. L. Cohen, Phys. Rev. B 32, 7988 (1985).

25. M.L. Cohen, Solid State Comm. 92, 45 (1994).

26. E. Knittle, R. M. Wentzcovitch, R. Jeanloz, and M. L. Cohen, Nature 337, 349 (1989).

27. A. Y. Liu and M. L. Cohen, Science 245, 841 (1989).

28. A.Y. Liu and R.M. Wentzcovitch, Phys. Rev. B 50, 10362 (1994).

29. K. M. Yu, M. L. Cohen, E. E. Haller, W. L. Hansen, A. Y. Liu, and I. C. Wu, Phys. Rev. B 49, 5034 (1994).

30. F. Fujimoto and K. Ogata, Jpn. J. Appl. Phys. 32, L420 (1993).

31. M.Y. Chen, D. Li, X. Lin, V.P. Dravid, Y.-W. Chung, M.-S. Wong, and W.D. Sproul, J. Vac. Sci. Techn. A 11 (13), 521 (1993).

32. C. Niu, Y.Z. Lu, and C. Lieber, Science 261, 334 (1993).

33. D: Li, X. Chu, S.-C. Cheng, X.-W. Lin, V.P. Dravid, Y.-W. Chung, M.-S. Wong, and W.D. Sproul, Appl. Phys. Lett. 67, 203 (1995).

34. H. Sjöström. S. Stafström, M. Boman, and J.-E. Sundgren, Phỵs. Rev. Lett. 75, 1336 (1995).

35. J. L. Corkill and M. L. Cohen, Phys. Rev. B 48, 17622 (1993).

36. H.W. Kroto, J.R. Heath, S.C. O'Brien, R.F. Curl, and R.E. Smalley, Nature 318, 162 (1985).

37. A.F. Hebard, M.J. Rosseinsky, R.C. Haddon, D.W. Murphy, S.H. Glarum, T.T.M. Palstra, A.P. Ramirez, and A.R. Kortan, Nature 350, 600 (1991).

38. E.L. Shirley and S.G. Louie, Phys. Rev. Lett. 71, 133 (1993).

39. M.L. Cohen, Phil. Mag. B70, 627 (1994).

40. W.L. McMillan, Phys. Rev. 167, 331 (1968).

41. O. Gunnarsson and G. Zwicknagl, Phys. Rev. Lett. 69,957 (1992). 
42. R.A. Jishi and M.S. Dresselhaus, Phys. Rev. B 45, 2597 (1992).

43. M. Schlüter, M. Lannoo, M. Needles, and G.A. Baraff, Phys. Rev. Lett. 68, 526 (1992).

44. C.M. Varma, J. Zaanen, and K. Raghavachari, Science 254, 989 (1991).

45. V. H. Crespi, J. G. Hou, X.-D. Xiang, M. L. Cohen, and A. Zettl, Phys. Rev. B 46, 12064 (1992).

46. S. Iijima, Nature 354,56 (1991).

47. N. Hamada, S. Sawada, and A. Oshiyama, Phys. Rev. Lett. 68, 1579 (1992).

48. Y. Miyamoto, A. Rubio, X. Blase, M.L. Cohen, and S.G. Louie, Phys. Rev. Lett. 74, 2993 (1995).

49. L.X. Benedict, V.H. Crespi, S.G. Louie, and M.L. Cohen, to be published.

50. A. Rubio, J. L. Corkill, and M. L. Cohen, Phys. Rev. B 49, 5081 (1994).

51. N.G. Chopra, F.J. Luyken, K. Cherri, V.H. Crespi, M.L. Cohen, S.G. Louie. and A. Zettl, Science 269, 966 (1995).

52. X. Blase, A. Rubio, S.G. Louie, and M.L. Cohen, Europhys. Lett. 28, 335 (1994).

53. Y. Miyamoto, A. Rubio, M. L. Cohen, and S. G. Louie, Phys. Rev. B 50, 4976 (1994).

54. Y. Miyamoto, A. Rubio, S.G. Louie, and M.L. Cohen, Phys. Rev. B 56, 18360 (1994).

55. L. Chico, V.H. Crespi, L.X. Benedict, M.L. Cohen, and S.G. Louie, to be published.

56. V.H. Crespi, L.X. Benedict, M.L. Cohen, and S.G. Louie, to be published.

57. C.H. Xu, G.Z. Wang, C.T. Chan, and K.M. Ho, J. Phys. Cond. Matter 4, 6047 (1992). 


\section{Figure Captions}

Figure 1: A heterostructure formed by the junction of a $(10,0)$ tube and a $(9,1)$ tube. The pentagonal-hexagonal defect in the center of the structure changes the tube indices by one unit, yielding a junction between semiconducting tubes of different band gaps. Related structures yield metal-semiconductor junctions.

Figure 2: The planar 5-7 carbon network. The primitive cell contains eight atoms and can be constructed from the atoms in the edge-sharing pentagons. 


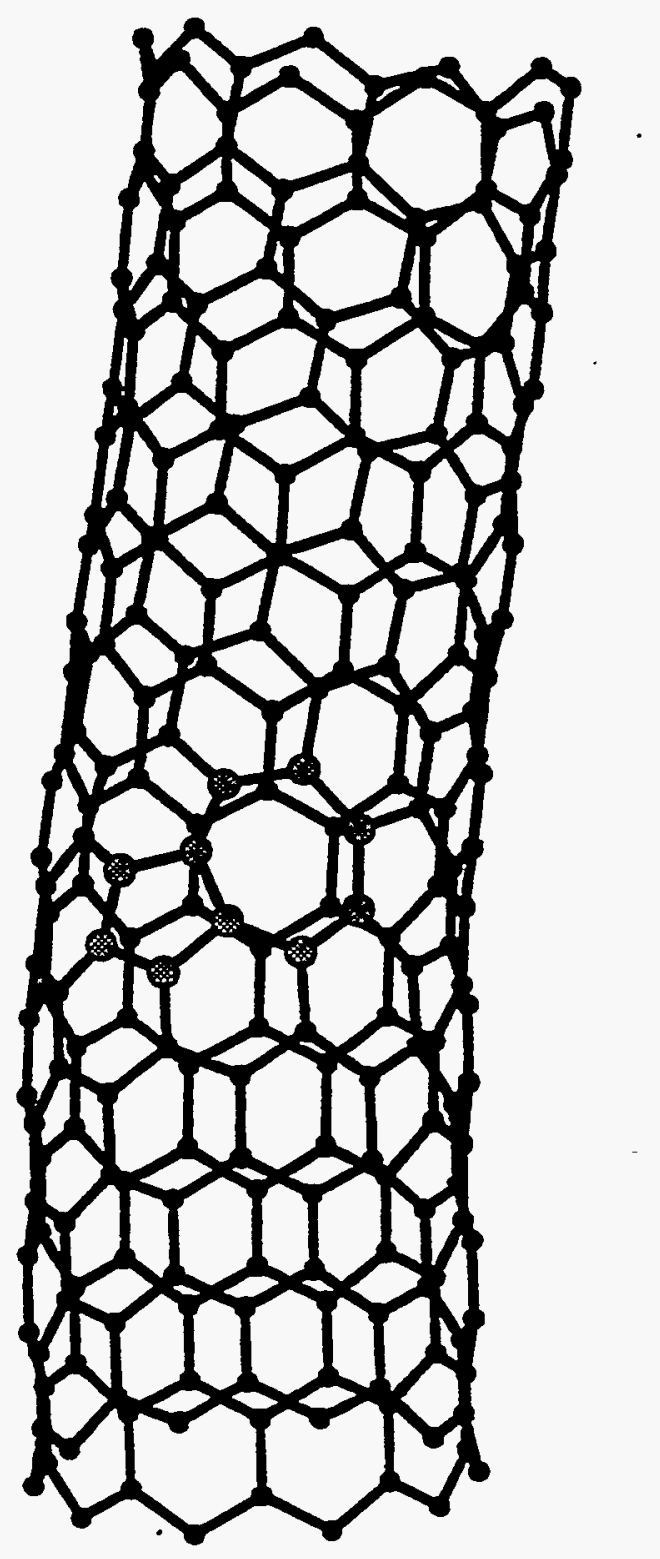

FIGURE 3 


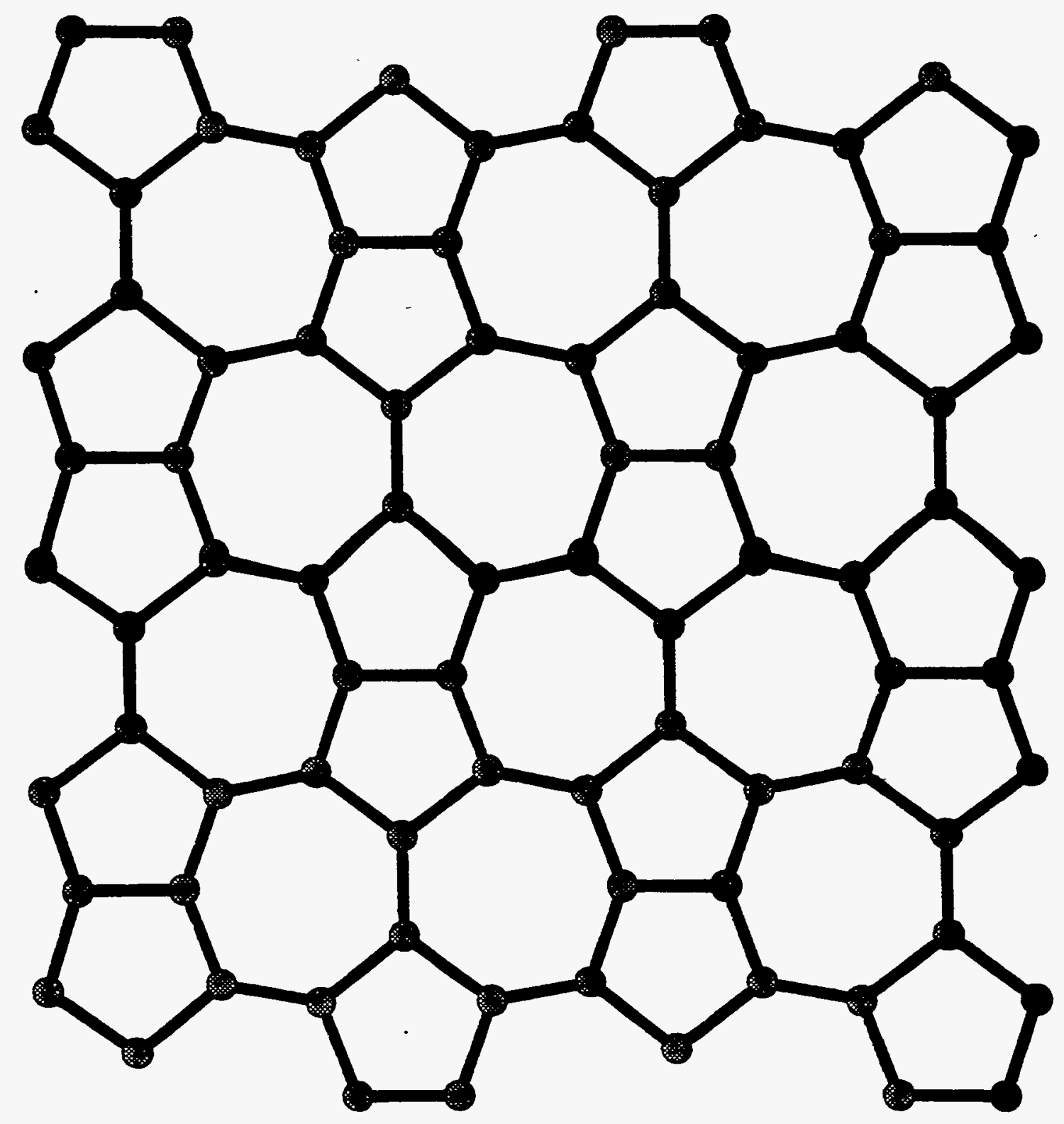

FIGURE 2 
